\title{
Clouds Toward the Virgo Cluster Periphery: Gas-rich Optically Inert Galaxies
}

\author{
Brian R. Kent ${ }^{1,2}$
}

\begin{abstract}
Aperture synthesis observations of two HI cloud complexes located in the periphery of the Virgo galaxy cluster are presented. These low HI-mass clouds $\left(M_{H I}<10^{9}\right)$ are seen projected on the $\mathrm{M}$ region of the western Virgo cluster, where the galaxy population is thought to lie behind the main A cluster surrounding M87. The kinematic measurements of both unresolved Arecibo and resolved VLA-C observations are in good agreement. The HI detections cannot be identified with any optical, IR, or UV emission from available archival imaging. They are inert at these wavelengths. The HI masses of the individual VLA detections range from $7.28 \leq \log \left(M_{H I}\right) \leq$ 7.85. The total dynamical mass estimates are several times their HI content, ranging from 7.00 $\leq \log \left(M_{d y n}\right) \leq 9.07$, with the assumption that the clouds are self-gravitating and in dynamical equilibrium. We report the observed parameters derived from the VLA observations. One of these HI clouds appears to be the most isolated optically inert detection observed in the outer reaches of Virgo.
\end{abstract}

Accepted for publication in The Astrophysical Journal.

Subject headings: galaxies: intergalactic medium — galaxies: halos — radio lines: galaxies — galaxies:clusters — galaxies: clusters: individual (Virgo)

\section{Introduction}

Galaxies are characterized by their stellar content, morphology, environment, dust, and neutral and molecular gas content. The $21 \mathrm{~cm}$ line of neutral hydrogen (HI) continues to play an important role in diagnosing the star formation potential of a galaxy and any past dynamic interactions with a cluster environment or neighboring galaxies. Blind HI surveys can sample the gas-rich population of the local Universe and search for lowmass, low-surface brightness systems.

The Arecibo Legacy Fast ALFA (ALFALFA; Giovanelli et al. 2005a) survey is providing such a sample of gas-rich objects. The project utilizes the seven-element Arecibo L-band Feed Array (ALFA) receiver system to conduct a wide area extragalactic HI investigation with the 305 meter Arecibo reflector. The survey improves over previous first generation surveys (HIPASS: Barnes et al. 2001, Meyer et al. 2004;
Wong et al. 2006; ADBS: Rosenberg \& Schneider 2002) in spectral and spatial resolution, providing $5 \mathrm{~km} \mathrm{~s}^{-1}$ resolution with a $3^{\prime}$ beam. The 7000 sq. deg. of surveyed sky covers a velocity range out to $c z_{\odot} \sim 18,000 \mathrm{~km} \mathrm{~s}^{-1}$. This includes interesting areas of the local supercluster such as the Virgo cluster at $c z_{\odot} \sim 1100 \mathrm{~km} \mathrm{~s}^{-1}$. The first and second ALFALFA Virgo catalogs (Giovanelli et al. 2007, Kent et al. 2008) comprise a complete mass limited dataset $\left(M_{H I} \geq 2 \times 10^{7} M_{\odot}\right.$ at Virgo). Sources identified in Kent et al. (2007; 2009) comprise a sample of optically inert HI detections; these objects are important in understanding the fate of galaxies in a cluster environment and its surrounding periphery. Previous detections that cannot be correlated with optical counterparts have been associated with nearby galaxy groups or nearby spiral galaxies harassed by the cluster environment (Ryder et al. 2001; Minchin et al. 2005; Oosterloo \& van Gorkom 2005; Haynes et al. 2007). 
Nearby galaxy cluster environments are of great interest to $\mathrm{HI}$ studies as both the gravitational potential and intra-cluster medium (ICM) perturb the gas structure and morphology of galaxies. Ram pressure stripping and tidal encounters at different cluster radii result in spiral galaxy deficiencies in HI of varying degrees. While threedimensional paths of galaxies through the cluster are often difficult to ascertain, the fingerprint of the cluster-galaxy interaction is well studied through aperture synthesis observations; high resolution studies show that $\mathrm{HI}$ radii are smaller than their optical counterparts and match predictions of theoretical studies (Giovanelli \& Haynes 1983).

Aperture synthesis observations have the ability to resolve higher sensitivity single dish survey detections. Resolved HI observations reveal the truncation of disks, tidal tails, and the disturbed morphology of late-type spiral galaxies (Cayatte et al. 1990,1994; Chung et al. 2006). All of these properties are indicators of galaxygalaxy and galaxy-cluster interactions. It has been shown that HI deficiency in late-type galaxies decreases with increasing cluster radius (Giovanelli $\&$ Haynes 1985). An important question of galaxy and cluster evolution that remains is: What happens to the stripped gas in the cluster environment? Will it be destroyed by ablation and evaporate into the cluster halo? Is it possible that an isolated cloud can survive and re-initiate star formation?

Here we present HI aperture synthesis observations of two HI clouds in the Virgo Cluster periphery, initially reported in Kent et al. (2007). These clouds, unresolved by Arecibo, are resolved into separate clumps with VLA-C observations. In section 2 we discuss the original Arecibo observations, data reduction, and detections. In section 3 we describe the follow-up VLA observations, data reduction, and detections. In section 4 we detail the environment and neighboring galaxies of the HI clouds. In section 5 we discuss possible cloud origins and compare to other gas-rich optically inert phenomena. Section 6 summarizes the results of the study.

\section{Single Dish Detections}

The HI clouds described here were detected by the ongoing ALFALFA survey. The meridian tran- sit observing strategy uses a sky drift mode with a $100 \mathrm{MHz}$ bandwidth and 4096 channels per polarization, centered at $1385 \mathrm{MHz}$. Each raw scan contains 14 spectra ( 7 beams $\times 2$ linear polarizations per beam), with a sampling rate of $1 \mathrm{~Hz}$ and spectral resolution of $24.4 \mathrm{kHz}\left(\delta V=5.1 \mathrm{~km} \mathrm{~s}^{-1}\right.$ at the rest frequency of the $21 \mathrm{~cm} \mathrm{HI}$ line). The system temperatures of the ALFA receivers during the observations were in the range $26 \mathrm{~K}<\mathrm{T}_{\text {sys }}<30 \mathrm{~K}$, yielding a root mean square (rms) noise of $\sigma_{m}=$ $2.5 \mathrm{mJy} /$ beam in channels with $\delta V=5.1 \mathrm{~km} \mathrm{~s}^{-1}$. The flagging, calibration, and gridding of the data into cubes are described in detail by Giovanelli et al. (2005a), Kent (2008) and Kent et al. (2009). Table 1 describes the parameters of the Arecibo observations.

The two HI clouds (henceforth Cloud 1 and 2) were detected in the ALFALFA data obtained in the Spring 2005 campaign sampling the Virgo cluster and its surrounding periphery. The detections were reported in Kent et al. (2007) as part of an initial collection of gas-rich, optically inert extragalactic objects. A complex of HI clouds situated halfway between M87 and M49 and their VLA observations were examined in Kent et al. (2009). Here we continue this effort with two HI clouds located in the $\mathrm{M}$ cloud periphery west of the main A cluster. Upon detection in the ALFALFA survey, both objects were re-observed and confirmed with the single pixel L-band Arecibo receiver. Integrated spectral profiles for each cloud are depicted in red in Figure 1]. The Arecibo observations show both sources as narrow, single peaked spectral profiles. Clouds 1 and 2 are unresolved by the Arecibo beam and are located $5.4^{\circ}(1.5 \mathrm{Mpc}$ in projection) and $4.2^{\circ}$ (1.2 $\mathrm{Mpc}$ in projection) from M87 respectively west toward the direction of the M cloud. The Arecibo detections are unresolved point sources and we cannot deduce any information about the morphology of the sources.

Table 2 describes the observed parameters and locations of these two HI detections as measured from the ALFALFA data cubes, derived in the manner described by Giovanelli et al. (2007). The spatial centroid of each cloud is in col. (2). Its accuracy depends on the source strength, and is of order $\sim 30^{\prime \prime}$ for the reported sources. The heliocentric velocity $c z_{\odot}$, width at $50 \%$ of the peak $W_{50}$ and total flux $F_{c}$ of the integrated spectral profiles in Figure 1 are in cols. (3)-(5). The signal- 
to-noise ratio $S / N$ of the detections is in col. (6), and is given by

$$
S / N=\left(\frac{1000 F_{c}}{W_{50}}\right) \frac{w_{s m o}^{1 / 2}}{\sigma_{r m s}}
$$

where $F_{c}$ is in $\mathrm{Jy} \mathrm{km} \mathrm{s}^{-1}, W_{50}$ is in $\mathrm{km} \mathrm{s}^{-1}, w_{s m o}$ is a smoothing width equal to the number of 10 $\mathrm{km} \mathrm{s}^{-1}$ bins bridging half the signal, and $\sigma_{r m s}$ is the rms noise (in mJy) across the integrated spectrum at $10 \mathrm{~km} \mathrm{~s}^{-1}$ resolution. The HI mass $M_{H I}$ for each cloud is in col. (7), and is computed assuming that the clouds are optically thin:

$$
M_{H I} / M_{\odot}=2.356 \times 10^{5} D^{2} F_{c},
$$

where $D$ is in Mpc and $F_{c}$ is in $\mathrm{Jy} \mathrm{km} \mathrm{s}{ }^{-1}$ (Roberts 1975). The uncertainties on $M_{H I}$ in Table 2 and elsewhere do not include errors in the distance adopted, which is poorly constrained due to the large peculiar velocities of objects near or within the cluster. As described in Giovanelli et al. (2005b) and Springob et al. (2006a), this results in ambiguities for galaxies with $c z_{\odot}<$ $3000 \mathrm{~km} \mathrm{~s}^{-1}$. The peculiar flow model used for the ALFALFA distances published in the catalogs corrects only for large-scale perturbations in the velocity field. The model is not able to deal effectively with regions in the immediate vicinity of Virgo. We adopt the same distance values for consistency with Kent et al. (2007): 16.7 Mpc for Cloud 1 and $34.8 \mathrm{Mpc}$ for Cloud 2. The model used to obtain the distances is based on the work of Tonry et al. (2000) and Masters et al. (2004), using a parametric model and spherical truncated power-law attractor to examine the peculiar motions that arise from a cluster like Virgo.

\section{Aperture Synthesis Follow-up Observa- tions}

Both HI cloud centroid positions were observed with the Very Large Array 3 in November 2006. Approximately nine hours of on-source integration were obtained in $\mathrm{C}$ configuration for each source. Online Hanning smoothing yielded a channel spacing of $12.2 \mathrm{kHz}$ over a bandpass of $1.5 \mathrm{MHz}$.

\footnotetext{
${ }^{3}$ The VLA is a facility of National Radio Astronomy Observatory, which is operated by Associated Universities, Inc., under a cooperative agreement with the National Science Foundation
}

The data from the runs were reduced using the Astronomical Image Processing System (AIPS; Greisen 2003) as described in Kent et al. (2009). Standard flux, phase and bandpass calibration and continuum subtraction routines were applied after flagging. The calibrated data were imaged using various weighting schemes; we analyze the highest sensitivity, naturally-weighted cubes with a synthesized beam width of $\sim 25^{\prime \prime}(\sim 2.0 \mathrm{kpc}$ at the Virgo distance). The data cubes created for the Cloud 1 field are not limited by dynamic range, and do not gain image fidelity from self-calibration. For the field with Cloud 2, self calibration was run using a strong continuum source (NVSS catalog position $\alpha=12^{h} 13^{m} 32.1^{s}$, $\delta=+13^{\circ} 07^{\prime} 20.4^{\prime \prime}$; Condon et al. 1998) of flux density $1.3 \mathrm{Jy}$, greatly improving the fidelity and phase calibration of the images. As part of the reduction process, each image was smoothed to the resolution of the ALFALFA data cubes to identify emission in channels, as well as their extent in the frequency domain. A summary of the aperture synthesis observing and map parameters is given in Table 3 For clarity, all variables denoting parameters derived from the VLA observations are primed.

Channel maps of each field are shown in Figure 2 and 3 with solid and dashed contours in the primary beam-corrected maps depicting, respectively, positive and negative multiples of the median rms map noise. All maps are corrected for the attenuation of the primary beam, and averaged over 3-4 spectral channels to yield a channel map resolution of $\delta V^{\prime}=7.8$ or $10.4 \mathrm{~km} \mathrm{~s}^{-1}$. The emission from detections in both fields is contiguous over multiple channels in different weighting and imaging deconvolution schemes.

Total intensity (zero moment) and intensityweighted velocity (first moment) maps of each field are shown in Figures 4 and 5 . The zero moment contours are overlayed on SDSS $g$-band images. For each frequency channel we blank regions with less than $3 \sigma_{m}^{\prime}$ (defined in Table 3) in the image before combining into the zero moment map. The first moment maps are computed only at locations with column densities of $N_{H I}^{\prime} \geq 1.5 \times 10^{20} \mathrm{~cm}^{-2}$ (Cloud 1) and $N_{H I}^{\prime} \geq 0.7 \times 10^{20} \mathrm{~cm}^{-2}$ (Cloud 2). Global integrated spectral profiles from all detections in each field, representing the total emission for comparison with the original ALFALFA spec- 
trum are shown in Figure 1 in blue. The rms error on the computed total emission over the full width half max (FWHM) range in each individual channel for both spectra range from 0.67 to $1.03 \mathrm{mJy}$ and also reflects a $5 \%$ uncertainty in calibration.

\subsection{HI Aperture Synthesis Detections}

Field for Cloud 1: We observe two detections which we label Cloud $1 \mathrm{~N}$ (North) and $1 \mathrm{~S}$ (South), the two brightest in the field. Individual integrated spectral profiles of each detection are shown in Figure 4. The northern cloud $1 \mathrm{~N}$ appears to be more aligned north-south whereas the lower signal-to-noise southern cloud $1 \mathrm{~S}$ is aligned east-west. The northern cloud $1 \mathrm{~N}$ exhibits a clear north-south velocity gradient. The velocity gradient of the southern cloud $1 \mathrm{~S}$ is slightly more disordered but trends along the east/west axis of the detection. There is no discernible structure on smaller scales or clumpiness as seen in the Virgo HI objects examined in Kent et al. (2009). No counterparts are observed in the field in any available optical, IR, or UV imaging databases. The combined kinematic properties of Clouds $1 \mathrm{~N}$ and $1 \mathrm{~S}$ agree well with the Arecibo data (Tables 2 and 4 ).

We recover $87 \pm 5 \%$ of the flux (Tables 2 and 4 ) from the Cloud 1 field in the VLA observations. The VLA global profile of all the integrated emission (which consists of Cloud $1 \mathrm{~N}$ and $1 \mathrm{~S}$ ) for $N_{H I}^{\prime} \geq 1.5 \times 10^{20} \mathrm{~cm}^{-2}$ in Figure 4 is shown in comparison with the Arecibo spectra in Figure 1. The mean velocity of Clouds $1 \mathrm{~N}$ and $1 \mathrm{~S}$ is 1229.5 $\mathrm{km} \mathrm{s}^{-1}$ and agrees with the centroid velocity of the Arecibo data. Some lower surface brightness HI might escape detection below the $3 \sigma_{m}^{\prime}$ level if it is distributed uniformly over the $30^{\prime \prime}$ region between the two detections. However, one would expect it to coincide kinematically with the mean velocity of the clouds.

Comparing the total $\mathrm{HI}$ mass of Clouds $1 \mathrm{~N}$ and $1 \mathrm{~S}$ from the VLA with that of Arecibo reveals little deficiency in the HI within the errors of the measurement (Tables 2 and 44). No detailed morphology can be ascertained from the VLA observations at the achieved sensitivity and spatial resolution, i.e. within each of the Clouds $1 \mathrm{~N}$ and $1 \mathrm{~S}$. This suggests that no flux exists on scales greater than $\sim 1^{\prime}$ and that any detectable structure of the detected blobs will be smaller than that spatial scale.
Field for Cloud 2: The emission detected in this field is, not unexpectedly, of lower signal-tonoise. The VLA global profile of all the integrated emission for $N_{H I}^{\prime} \geq 0.7 \times 10^{20} \mathrm{~cm}^{-2}$ in Figure 5] is shown in comparison with the Arecibo spectra in Figure 11. We recover $41 \pm 5 \%$ of the flux (Tables 2 and (4) compared to the Arecibo data. It is likely that lower column density emission is resolved out and we are only detecting the higher column density peaks of the source. We identify three of these peaks (Cloud 2 North, West, and South) fit for the same parameters as the Cloud 1 field. Clouds 2 North and West are elongated in an east-west direction, where the Southern component is a marginal detection in the map with low signal to noise, albeit still a $5 \sigma_{m}^{\prime}$ detection in the map. The velocity maps of all three Cloud 2 field detections show no ordered motion. The clutter in the field contains a number of bumps one or two sigma above the rms noise.

\subsection{Field Properties}

The properties for the detections in both fields are summarized in Table 4 in the same manner as Kent et al. (2009). The global emission parameters, where measurable, are also listed. Each detected feature in the VLA data cubes was fit with a centroid ellipse in the same fashion as the Arecibo data. The centroid positions of these fits for each individual cloud detection in the total intensity maps of Field 1 and 2 (Figures 4 and 5) are given in col. (2). The centroid $c z_{\odot}^{\prime}$ of both the individual integrated profiles of Figure 6 is in col. (3), and $W_{50}^{\prime}$ of the profiles is in col. (4). The values of $W_{50}^{\prime}$ are corrected for instrumental effects by assuming that the unbroadened profile is Gaussian. The integrated flux density $F_{c}^{\prime}$ and HI mass $M_{H I}^{\prime}$ are in cols. (5) and (8), respectively. The major axis $a_{H I}^{\prime}$ measured from the ellipsoidal fits of each individual cloud is in col. (6). We adopt the outermost locations as the edges of the clouds where $N_{H I}^{\prime}=1.5 \times 10^{20} \mathrm{~cm}^{-2}$ (Figure 4) and $N_{H I}^{\prime}=0.7 \times 10^{20} \mathrm{~cm}^{-2}$ (Figure 5). The position angle $P A_{H I}^{\prime}$ at which $a_{H I}^{\prime}$ is measured is in col. (7). An estimate of the dynamical mass $M_{d y n}^{\prime}$ of each cloud is in col. (9), and is computed via:

$$
M_{d y n}^{\prime}=\left(3.39 \times 10^{4}\right) a_{H I}^{\prime} D\left(\frac{W_{50}^{\prime}}{2}\right)^{2},
$$


where $a_{H I}^{\prime}$ is the object diameter in arcminutes, $W_{50}^{\prime}$ is in $\mathrm{km} \mathrm{s}^{-1}$ and the distance $D$ is in Mpc. We note that $M_{d y n}^{\prime}$ has physical meaning only if the clouds are self-gravitating and in dynamical equilibrium, which may or may not be a valid assumption.

\section{The Environment of the Cloud Com- plexes}

Figure 7 shows the location of these two HI clouds with respect to the Virgo Cluster, with the boundaries and areas of Binggeli et al. (1993) and plotted against the hot X-ray cluster background detected by ROSAT (Snowden et al. 1995). Both cloud regions are also outside the projected virial radius of the dark matter halo around M87 determined by McLaughlin (1999). The detections lie in the vicinity of the $\mathrm{M}$ cloud (Ftaclas et al. 1984) west of M87, where member galaxies are considered to lie behind the main A cluster. These $\mathrm{M}$ cloud galaxies have a larger mean velocity $\left(c z_{\odot} \sim 2000 \mathrm{~km} \mathrm{~s}^{-1}\right)$ than the main cluster $\left(c z_{\odot} \sim 1150 \mathrm{~km} \mathrm{~s}^{-1}\right)$. However, peculiar velocities due to the large central mass of the cluster yield a velocity dispersion of the projected $\mathrm{M}$ cloud region galaxies that ranges from $-100<c z_{\odot}<$ $2400 \mathrm{~km} \mathrm{~s}^{-1}$. The assignment of these HI features to the various areas of Virgo remains ambiguous.

Imaging studies by Roberts et al. (2007) analyzed an area extending from the eastern part of the $\mathrm{M}$ cloud northward. They arrived at a density of 20 to 60 low surface brightness (LSB) dwarf galaxies per square degree in the area $1.5^{\circ}$ west of M87. This may have some relation to possible parent galaxies of the Cloud 2 field; Figure 7 shows that the Cloud 1 field is more removed from the main cluster and this density measurement.

No obvious optical features that resemble an extragalactic counterpart can be correlated with any of the cloud components in online imaging databases. The catalogs provided by the NASA/IPAC Extragalactic Database (NED), Sloan Digital Sky Survey (York et al. 2000), Virgo Cluster Catalog (Binggeli et al. 1985), GOLDMine (Gavazzi et al. 2003) and published ALFALFA survey (Giovanelli et al. 2008; Kent et al. 2009) were examined for possible nearby associations to the Cloud 1 and 2 fields. Figure 8 shows $2^{\circ} \times 2^{\circ}$ areas of sky surrounding each of the Arecibo detections. Each plot contains open circles from galaxies with published HI or optical redshifts within a given range of the measured Arecibo/HI velocity for the detection; $c z_{\odot} \leq 3000 \mathrm{~km} \mathrm{~s}^{-1}$ for the Cloud 1 area and $1400<c z_{\odot}<3000 \mathrm{~km} \mathrm{~s}^{-1}$ for the Cloud 2 area. Galaxies in those areas with no published redshifts at any wavelength are depicted as small crosses. We next examine a number of nearby galaxies of comparable redshift within the projected vicinity of Cloud 1 and Cloud 2.

\subsection{The Projected Environment of Cloud 1}

The galaxy environment below a redshift of $c z_{\odot} \leq 3000 \mathrm{~km} \mathrm{~s}^{-1}$ is rather sparse in the vicinity of Cloud 1. Galaxies that meet this criteria are listed in Table 5. Of particular note is the faint galaxy SDSS J120859.92+115631.2. This faint detection lies 3.8 $\mathrm{NE}$ of the Arecibo centroid. It is the closest optical detection near Cloud 1 ; it remains ambiguous as to whether this object is a Virgo Cluster member or a more distance background galaxy. The SBb(r)I-II galaxy VCC 58 has a disturbed morphology and optical redshift of $c z_{\odot}=2188 \mathrm{~km} \mathrm{~s}^{-1}$ (Rubin et al. 1999) and $\mathrm{HI}$ redshift of $c z_{\odot}=2209 \mathrm{~km} \mathrm{~s}^{-1}$ (Giovanelli et al. 2007). This is the only spiral galaxy within $\sim 1^{\circ}$ though its velocity differs by $\sim 1000 \mathrm{~km} \mathrm{~s}^{-1}$. It is described as having a disturbed rotation curve (Rubin et al. 1999).

\subsection{The Projected Environment of Cloud 2}

Cloud 2 lies in a much higher galaxy density environment and includes, within $2^{\circ}$, nine galaxies with a cataloged late type morphology for 1400 $<c z_{\odot}<3000 \mathrm{~km} \mathrm{~s}^{-1}$ (Table 6). Several galaxies have notable relevant properties. The aforementioned VCC 58 lies $49^{\prime}$ southwest of Cloud 2 and is of comparable redshift. The closest published HI detection is VCC 85, detected by Gavazzi et al. (2006) with $c z_{\odot}=1932 \mathrm{~km} \mathrm{~s}^{-1}$ and lies $\sim 8^{\prime}$ from the Cloud 2 centroid. Also of note is VCC 97, an SAB galaxy that lies $17^{\prime}$ North of Cloud 2. Chamaraux et al. (1980) showed this galaxy to be HI deficient $\left(\mathrm{DEF}_{H I}=0.21\right.$; Helou, Hoffmann, \& Salpeter 1984); it has an HI redshift of $c z_{\odot}=2470 \mathrm{~km} \mathrm{~s}^{-1}$. Doyon \& Joseph (1989) also noted a dust deficiency in VCC 97. 


\section{Discussion}

Roberts (1988; see references therein) outlined various categories of intergalactic HI clouds: tidal tails, extended HI envelopes, and clouds near groups or within clusters. Starless intergalactic clouds in the field, isolated from other galaxies, have yet to be detected. Recent studies have focused on the search and identification of starless gas-rich halos. As one of the many important science goals, identifying such objects in blind surveys like HIPASS and ALFALFA give useful information on the formation and evolution of galaxies in a variety of environments. Recently detected optically inert clouds and their respective followup studies can be associated with galaxies in nearby clusters (Sancisi et al. 1987; Davies et al. 2004; Minchin et al. 2005; Haynes et al. 2007; Kent et al. 2007), in groups or disturbed galaxies (Schneider et al. 1983; Henning et al. 1993; Ryder et al. 2001), in tidal or harassed tails (Oosterloo \& van Gorkom 2005; Giovanelli \& Haynes 1989; Salzer et al. 1991), or as an HVC or Milky Way/Local Group companion (Kilborn et al. 2000; Giovanelli et al. 2010). However, surveys have not revealed a large population of previously undetected dark matter halos predicted by large scale simulations (Moore et al. 1999). In the nearby Virgo Cluster, the HI detected in clouds or tidal streams does not make up a significant portion of the HI deficiency in nearby parent spirals; the population of HI clouds does not, by itself, offer a complete solution to the missing satellite or mass problem (Kent et al. 2009; Klypin et al. 1999).

None of the detections discussed here appear to be tidal tails that clearly extend to an obvious parent galaxy. The largest tail extending from a Virgo galaxy is near NGC 4532 at a length of 500 kpc (Koopmann et al. 2008). While late type spirals and dwarfs are within a projected $500 \mathrm{kpc}$ range of both Cloud 1 and Cloud 2, neither has a tail or streamlike morphology leading to another nearby galaxy. The clouds do not belong to a compact group of galaxies, nor are they are part of the main A or B clusters surrounding M87 or M49.

Although the region surrounding the Cloud 1 detection lies at a projected distance of $1.5 \mathrm{Mpc}$ from M87, it has been shown that the spiral galaxy population in the $\mathrm{M}$ cloud area is $\mathrm{HI}$ deficient. The
Cloud 1 detection lies on the boundary of higher HI deficiency (Solanes et al. 2001), whereas the Cloud 2 field lies within it. The intracluster X-ray density in the vicinity of the Virgo M cloud is estimated to be $n_{i \mathrm{~cm}} \sim 3 \times 10^{-6} \mathrm{~cm}^{-3}$ (computed from Vollmer et al. 2001). The Virgo Cluster ICM temperature maps computed by Shibata et al. (2001) do not cover the region of sky containing the HI clouds. If we entertain the assumption that these clouds came from a spiral disk, then the presence of this gas deficiency means that a ram pressure stripping hypothesis cannot be completely discarded.

We can place upper limits on the optical surface brightness based on models of Bell et al. (2003). As in Kent et al. (2009) we assume a $g$-band imaging surface brightness limit similar to other SDSS LSB galaxy studies $\left(\mu_{g} \sim 26\right.$ mag $\operatorname{arcsec}^{-2}$; Kniazev et al. 2004). A feature of source size $\sim 10^{\prime \prime}$ would have a $g$-band luminosity of $L_{g} \sim 10^{6} L_{\odot}$ and model stellar M/L ratio of $M^{*} / L^{*} \sim 1.6$. The theoretical upper limits for the stellar to $\mathrm{HI}$ mass ratio would range from $\sim 0.02$ to 0.11 for the clouds extracted from the VLA data cubes. Upper limits for the HI mass to stellar luminosity would range from $\sim 15$ to 70 . It remains an open issue as to whether or not any optical emission can be positively correlated with these HI detections.

As indicated in Section 4, nine late-type galaxies lie in the vicinity and near redshift range of Cloud 2; only one lies $\sim 1^{\circ}$ Northeast of Cloud 1. This makes it difficult to identify a parent galaxy. However, we can hypothetically consider the movement of these clouds through the cluster environment. As both clouds are at higher velocities than the systemic heliocentric cluster velocity $\left(c z_{\odot, \text { Virgo }} \sim 1150 ; \mathrm{km} \mathrm{s}^{-1}\right.$ Huchra 1988), their line-of-sight velocity with respect to the cluster reference frame is directed away from us. If the clouds were torn from a spiral disk that is in a similar reference frame, with the clouds decelerating, then the parent galaxy would be at a higher systemic velocity than the cluster. The only nearby spiral galaxy of comparable velocity is the aforementioned VCC 58 (IC 769), located one degree Northeast of the Cloud 1 detection at a redshift $c z_{\odot}=2209 \mathrm{~km} \mathrm{~s}^{-1}$. VCC 58 also lies one degree Southwest of Cloud 2, and stands as a remote, yet possible candidate parent of either cloud. 


\section{Summary}

We have presented new follow-up observations obtained with the Very Large Array that resolve original Arecibo HI detections of extragalactic HI clouds in the Virgo Cluster periphery. The results of these observations are summarized as follows:

1. Two HI clouds detected and unresolved with Arecibo using ALFALFA survey data. The HI detections have heliocentric radial velocities of $c z_{\odot}=1230$ and $2235 \mathrm{~km} \mathrm{~s}^{-1}$. The velocity widths are narrow at 29 and $53 \mathrm{~km} \mathrm{~s}^{-1}$. The HI masses of Cloud 1 and 2 are, respectively, $4.3 \times 10^{7}$ and $3.5 \times 10^{8} M_{\odot}$.

2. Detections have been made with the VLA in both the Cloud 1 and Cloud 2 fields at the same velocities as the Arecibo detections. The data show two and three separated regions of $\mathrm{HI}$ emission for the Cloud 1 and Cloud 2 fields respectively. The individual $\mathrm{HI}$ masses range from $\log \left(M_{H I}^{\prime}\right)=7.1-$ $7.8 M_{\odot}$. We recover $87 \%$ of the flux for the Cloud 1 field and $41 \%$ of the flux for the Cloud 2 field. No optical, IR, or UV counterpart can be identified with these HI features using available online imaging databases.

3. The galaxy environment is relatively sparse around Cloud 1 - one faint object with no redshift information, SDSS J120859.92+115631.2, lies $3.8^{\prime}$ northeast of the Arecibo centroid. The nearest late-type galaxy of comparable Virgo redshift is VCC 58, located one degree to the northeast.

4. The Cloud 2 detection lies in a dense galaxy environment showing higher HI deficiency with nine late type spiral systems of comparable Virgo redshift within a one degree radius. The closest HI detection is VCC 85 at $8^{\prime}$.

5. The HI deficient spirals in the M cloud region show that dynamic processes are prevalent even at large distances from the Virgo Cluster center. While there are no larger spirals immediately in the vicinity or at comparable velocity of the HI Clouds, we cannot dismiss a cloud origin hypothesis of ram pressure stripping. Much like previous detections reported in Kent et al. (2007;
2009), it is unlikely that the HI clouds described here are primordial gas structures in dark matter halos. These two clouds are located in the outer parts of the cluster and are in a lower density environment than other $\mathrm{HI}$ clouds and tidal tails further toward M87 or M49. Cloud 1 remains unique in its isolation. To date, there are no other gas structures that are both definitively extragalactic and unambiguously not associated with another galaxy outside the Local Group.

We wish to thank David E. Hogg and Morton S. Roberts of the NRAO for their assistance and encouragement of this work. We also wish to thank William Cotton for advice on imaging techniques, and the anonymous referee for their careful review.

BRK acknowledges support from a Jansky Fellowship during the completion of this work. This research has made use of the NASA/IPAC Extragalactic Database (NED) which is operated by the Jet Propulsion Laboratory, California Institute of Technology, under contract with the National Aeronautics and Space Administration. Skyview was developed and maintained under NASA ADP Grant NAS5-32068 under the auspices of the High Energy Astrophysics Science Archive Research Center at the Goddard Space Flight Center Laboratory of NASA.

This research has made use of Sloan Digital Sky Survey (SDSS) data. Funding for the SDSS has been provided by the Alfred P. Sloan Foundation, the Participating Institutions, the National Aeronautics and Space Administration, the National Science Foundation, the U.S. Department of Energy, the Japanese Monbukagakusho, and the Max Planck Society. The SDSS Web site is http://www.sdss.org/. The SDSS is managed by the Astrophysical Research Consortium (ARC) for the Participating Institutions. The Participating Institutions are The University of Chicago, Fermilab, the Institute for Advanced Study, the Japan Participation Group, The Johns Hopkins University, the Korean Scientist Group, Los Alamos National Laboratory, the Max-Planck-Institute for Astronomy (MPIA), the Max-Planck-Institute for Astrophysics (MPA), New Mexico State University, University of Pittsburgh, University of Portsmouth, Princeton University, the United States Naval Observatory, and the University of Washington. 


\section{REFERENCES}

Adelman-McCarthy, J. K., et al. 2006, ApJS, 162, 38

Barnes, D. G., et al. 2001, MNRAS, 322, 486

Bell, E. F., McIntosh, D. H., Katz, N., \& Weinberg, M. D. 2003, ApJS, 149, 289

Binggeli, B., Popescu, C. C., \& Tammann, G. A. 1993, A\&AS, 98, 275

Binggeli, B., Sandage, A., \& Tammann, G. A. 1985, AJ, 90, 1681

Cayatte, V., Kotanyi, C., Balkowski, C., \& van Gorkom, J. H. 1994, AJ, 107, 1003

Cayatte, V., van Gorkom, J. H., Balkowski, C., \& Kotanyi, C. 1990, AJ, 100, 604

Chamaraux, P., Balkowski, C., \& Gerard, E. 1980, A\&A, 83, 38

Chung, A., van Gorkom, J. H., Kenney, J. D. P., \& Vollmer, B. 2007, ApJL, 659, L115

Condon, J. J., Cotton, W. D., Greisen, E. W., Yin, Q. F., Perley, R. A., Taylor, G. B., \& Broderick, J. J. 1998, AJ, 115, 1693

Davies, J., et al. 2004, MNRAS, 349, 922

Doyon, R., \& Joseph, R. D. 1989, MNRAS, 239, 347

Falco, E. E., et al. 1999, PASP, 111, 438

Fisher, K. B., Huchra, J. P., Strauss, M. A., Davis, M., Yahil, A., \& Schlegel, D. 1995, ApJS, 100,69

Ftaclas, C., Struble, M. F., \& Fanelli, M. N. 1984, ApJ, 282, 19

Gavazzi, G., Boselli, A., Donati, A., Franzetti, P., \& Scodeggio, M. 2003, A\&A, 400, 451

Gavazzi, G., O’Neil, K., Boselli, A., \& van Driel, W. 2006, A\&A, 449, 929

Giovanelli, R., \& Haynes, M. P. 1985, ApJ, 292, 404

Giovanelli, R., \& Haynes, M. P. 1983, AJ, 88, 881

Giovanelli, R., \& Haynes, M. P. 1989, ApJL, 346, L5

Giovanelli, R., et al. 2005a, AJ, 130, 2613

Giovanelli, R., et al. 2005b, AJ, 130, 2598

Giovanelli, R., et al. 2007, AJ, 133, 2569

Greisen, E. W. 2003, Astrophysics and Space Science Library, 285, 109

Haynes, M. P., Giovanelli, R., \& Kent, B. R. 2007, ApJL, 665, L19

Helou, G., Hoffman, G. L., \& Salpeter, E. E. 1984, ApJS, 55, 433

Henning, P. A., Sancisi, R., \& McNamara, B. R. 1993, A\&A, 268, 536

Kent, B. R., et al. 2008, AJ, 136, 713

Kent, B. R., et al. 2007, ApJL, 665, L15

Kent, B. R., Spekkens, K., Giovanelli, R., Haynes, M. P., Momjian, E., Cortés, J. R., Hardy, E., \& West, A. A. 2009 , ApJ, 691 , 1595

Kent, B. R. 2008, Ph.D. Thesis, Cornell University

Kilborn, V. A., et al. 2000, AJ, 120, 1342

Klypin, A., Kravtsov, A. V., Valenzuela, O., \& Prada, F. 1999, ApJ, 522, 82

Kniazev, A. Y., Grebel, E. K., Pustilnik, S. A., Pramskij, A. G., Kniazeva, T. F., Prada, F., \& Harbeck, D. 2004, AJ, 127, 704

Koopmann, R. A., et al. 2008, ApJL, 682, L85

Masters, K. L., Haynes, M. P., \& Giovanelli, R. 2004, ApJL, 607, L115

McLaughlin, D. E. 1999, ApJL, 512, L9

Meyer, M. J., et al. 2004, MNRAS, 350, 1195 
Minchin, R., et al. 2005, ApJL, 622, L21

Moore, B., Ghigna, S., Governato, F., Lake, G., Quinn, T., Stadel, J., \& Tozzi, P. 1999, ApJL, 524, L19

Oosterloo, T., \& van Gorkom, J. 2005, A\&A, 437, L19

Roberts, M. S. 1988, New Ideas in Astronomy, 65

Roberts, M. S. 1975, Galaxies and the Universe, 309

Roberts, S., Davies, J., Sabatini, S., Auld, R., \& Smith, R. 2007, MNRAS, 379, 1053

Rosenberg, J. L., \& Schneider, S. E. 2002, ApJ, 567, 247

Rubin, V. C., Waterman, A. H., \& Kenney, J. D. P. 1999, AJ, 118, 236

Ryder, S. D., et al. 2001, ApJ, 555, 232

Salzer, J. J., di Serego Alighieri, S., Matteucci, F., Giovanelli, R., \& Haynes, M. P. 1991, AJ, 101, 1258

Sancisi, R., Thonnard, N., \& Ekers, R. D. 1987, ApJL, 315, L39

Schneider, S. E., Helou, G., Salpeter, E. E., \& Terzian, Y. 1983, ApJL, 273, L1

Shibata, R., Matsushita, K., Yamasaki, N. Y., Ohashi, T., Ishida, M., Kikuchi, K., Böhringer, H., \& Matsumoto, H. 2001, ApJ, 549, 228

Snowden, S. L., et al. 1995, ApJ, 454, 643

Solanes, J. M., Manrique, A., García-Gómez, C., González-Casado, G., Giovanelli, R., \& Haynes, M. P. 2001, ApJ, 548, 97

Springob, C. M., Haynes, M. P., \& Giovanelli, R. 2005, ApJ, 621, 215

Springob, C. M., Haynes, M. P., Giovanelli, R., \& Kent, B. R. 2005, ApJS, 160, 149

Tonry, J. L., Blakeslee, J. P., Ajhar, E. A., \& Dressler, A. 2000, ApJ, 530, 625

Tully, R. B., Shaya, E. J., Karachentsev, I. D., Courtois, H. M., Kocevski, D. D., Rizzi, L., \& Peel, A. 2008, ApJ, 676, 184

Vollmer, B., Cayatte, V., Balkowski, C., \& Duschl, W. J. 2001, ApJ, 561, 708

Wong, O. I., et al. 2006, MNRAS, 371, 1855

York, D. G., et al. 2000, AJ, 120, 1579

This 2-column preprint was prepared with the AAS IATEX macros v5.0. 
TABLE 1

AlfAlfA Observing and Data Cube Parameters

\begin{tabular}{lc}
\hline \hline \multicolumn{1}{c}{ Parameter } & Value \\
\hline Spectral range & $25 \mathrm{MHz}\left(-2000-3200 \mathrm{~km} \mathrm{~s}^{-1}\right)$ \\
Effective integration time & 48 seconds $(\text { beam solid angle })^{-1}$ \\
Spectral resolution $\delta V$ & $24.4 \mathrm{kHz}\left(5.1 \mathrm{~km} \mathrm{~s}^{-1}\right)$ \\
Half-power beam size & $3^{\prime} .3 \times 3^{\prime} .8$ \\
RMS noise $\sigma_{m}$ for $\delta V=5.1 \mathrm{~km} \mathrm{~s}^{-1}$ & $2.5 \mathrm{mJy} /$ beam \\
\hline
\end{tabular}

TABLE 2

Arecibo Single-Dish Cloud Properties

\begin{tabular}{ccccccc}
\hline \hline Cloud & $\begin{array}{c}\alpha, \delta \\
(\mathrm{J} 2000) \\
(2)\end{array}$ & $\begin{array}{c}c z_{\odot} \\
\left(\mathrm{km} \mathrm{s}^{-1}\right) \\
(3)\end{array}$ & $\begin{array}{c}W_{50} \\
\left(\mathrm{~km} \mathrm{~s}^{-1}\right) \\
(4)\end{array}$ & $\begin{array}{c}F_{c} \\
\left(\mathrm{Jy} \mathrm{km} \mathrm{s}^{-1}\right)\end{array}$ & $\begin{array}{c}S / N \\
(5)\end{array}$ & $\begin{array}{c}\log \left(M_{H I} / M_{\odot}\right) \\
(6)\end{array}$ \\
\hline Cloud 1 & $120845.5,+115517$ & $1230 \pm 1$ & $29 \pm 2$ & $0.77 \pm 0.04$ & 11.6 & 7.63 \\
Cloud 2 & $121341.8,+125351$ & $2235 \pm 2$ & $53 \pm 3$ & $1.21 \pm 0.07$ & 9.2 & 8.54 \\
\hline
\end{tabular}

Note.-Col. (1): cloud name. Col. (2): right ascension and declination of cloud centroid (J2000). Col. (3): average heliocentric velocity of integrated spectral profile from Figure 1 Col. (4): profile width, measured at $50 \%$ of the integrated spectra profile peak and corrected for instrumental broadening as described in Giovanelli et al. (2007). Col. (5): total flux of integrated spectral profile. Col. (6): signalto-noise ratio of the detection, computed using $W_{50}$ and $F_{c}$ via eq. 1 Col. (7): base 10 logarithm of total HI mass, computed using $F_{c}$ via eq. 2

TABLE 3

Aperture Synthesis Observing and Data Cube Parameters

\begin{tabular}{lcc}
\hline \hline \multicolumn{1}{c}{ Parameter } & Cloud 1 & Cloud 2 \\
\hline Pointing center (J2000) & $12^{h} 08^{m} 45.5^{s},+11^{\circ} 55^{\prime} 17^{\prime \prime}$ & $12^{h} 13^{m} 41.8^{s},+12^{\circ} 53^{\prime} 51^{\prime \prime}$ \\
Total time on-source & 547 minutes & $532 \mathrm{minutes}$ \\
Net bandpass & $1.5 \mathrm{MHz}\left(1132-1336 \mathrm{~km} \mathrm{~s}^{-1}\right)$ & $1.5 \mathrm{MHz}\left(2137-2341 \mathrm{~km} \mathrm{~s}{ }^{-1}\right)$ \\
Maximum spectral resolution $\delta V^{\prime}$ & $12.2 \mathrm{kHz}\left(2.6 \mathrm{~km} \mathrm{~s}^{-1}\right)$ & $12.2 \mathrm{kHz}\left(2.6 \mathrm{~km} \mathrm{~s}^{-1}\right)$ \\
Synthesized beam / natural weighting & $25.2^{\prime \prime} \times 24.4^{\prime \prime} @ 56.7^{\circ}$ & $25.3^{\prime \prime} \times 24.4^{\prime \prime} @ 57.2^{\circ}$ \\
$\sigma_{m}^{\prime}$ at pointing center, $\delta V^{\prime}=2.6 \mathrm{~km} \mathrm{~s}^{-1}$ & $1.39 \mathrm{mJy} / \mathrm{beam}$ & $1.37 \mathrm{mJy} / \mathrm{beam}$ \\
\hline
\end{tabular}


TABLE 4

Aperture Synthesis Cloud Properties from the Vla Observations

\begin{tabular}{|c|c|c|c|c|c|c|c|c|}
\hline $\begin{array}{c}\text { Feature } \\
\text { (1) }\end{array}$ & $\begin{array}{c}(\alpha, \delta)^{\prime} \\
(\mathrm{J} 2000) \\
(2)\end{array}$ & $\begin{array}{c}c z_{\odot}^{\prime} \\
\left(\mathrm{km} \mathrm{s}^{-1}\right) \\
(3)\end{array}$ & $\begin{array}{c}W_{50}^{\prime} \\
\left(\mathrm{km} \mathrm{s}^{-1}\right) \\
(4)\end{array}$ & $\begin{array}{c}F_{c}^{\prime} \\
\left(\mathrm{Jy} \mathrm{km} \mathrm{s}^{-1}\right) \\
(5)\end{array}$ & $\begin{array}{l}a_{H I}^{\prime} \\
\left({ }^{\prime}\right) \\
(6)\end{array}$ & $\begin{array}{c}P A_{H I}^{\prime} \\
\left({ }^{\circ}\right) \\
(7)\end{array}$ & $\begin{array}{c}\log \left(M_{H I}^{\prime} / M_{\odot}\right) \\
(8)\end{array}$ & $\begin{array}{c}\log \left(M_{d y n}^{\prime} / M_{\odot}\right) \\
(9)\end{array}$ \\
\hline Cloud 1 Global & - & $1229 \pm 2$ & $26 \pm 4$ & $0.67 \pm 0.03$ & - & - & 7.64 & - \\
\hline Cloud 1 North & $120847.6,+115557$ & $1234 \pm 3$ & $22 \pm 6$ & $0.29 \pm 0.03$ & $1.0 \pm 0.3$ & -268 & 7.28 & 7.83 \\
\hline Cloud 1 South & $120847.4,+115448$ & $1225 \pm 3$ & $20 \pm 8$ & $0.39 \pm 0.03$ & $1.4 \pm 0.3$ & 13 & 7.40 & 7.65 \\
\hline Cloud 2 Global & - & $2231 \pm 4$ & $51 \pm 6$ & $0.50 \pm 0.02$ & - & - & 8.15 & - \\
\hline Cloud 2 North & $121342.5,+125450$ & $2237 \pm 2$ & $13 \pm 4$ & $0.14 \pm 0.02$ & $2.5 \pm 0.04$ & -7 & 7.60 & 8.09 \\
\hline Cloud 2 West & $121333.1,+125244$ & $2205 \pm 5$ & $41 \pm 9$ & $0.25 \pm 0.02$ & $2.4 \pm 0.03$ & -4 & 7.85 & 9.07 \\
\hline Cloud 2 South & $121341.9,+125116$ & $2234 \pm 3$ & $6 \pm 5$ & $0.05 \pm 0.01$ & $0.8 \pm 0.02$ & 39 & 7.15 & 7.00 \\
\hline
\end{tabular}

Note.-Col. (1): Cloud identifier. Col. (2): centroid RA and Decl. based on the fitting of ellipses to each detection. Col. (3): average heliocentric velocity of integrated spectral profile from Figure 6] Col. (4): profile width, measured at $50 \%$ of the integrated spectral profile peak and corrected for instrumental effects assuming that unbroadened profile is Gaussian. Col. (5): total flux of integrated spectral profile. Col. (6): maximum linear extent of region with $N_{H I}^{\prime} \geq 1.5 \times 10^{20} \mathrm{~cm}^{-2}$ (Cloud 1 field) and $N_{H I}^{\prime} \geq 0.7 \times 10^{20} \mathrm{~cm}^{-2}$ (Cloud 2 field) in the total intensity maps (Figs 4 and 5 ). Col. (7): position angle at which $a_{H I}^{\prime}$ was measured. Col. (8): base 10 logarithm of total H I mass, computed using $F_{c}^{\prime}$ via Equation 2 Col. (9): base 10 logarithm of the dynamical mass, computed using $W_{50}^{\prime}$ and $a_{H I}^{\prime}$ via Equation 3

TABLE 5

Galaxy Environment of Cloud $1\left(c z_{\odot}=1230 \mathrm{~km} \mathrm{~s}^{-1}\right)$

\begin{tabular}{|c|c|c|c|c|c|c|c|}
\hline $\begin{array}{c}\text { Galaxy Name } \\
(1)\end{array}$ & $\begin{array}{c}(\alpha, \delta) \\
(\mathrm{J} 2000) \\
(2)\end{array}$ & $\begin{array}{c}c z_{\odot, H I} \\
\left(\mathrm{~km} \mathrm{~s}^{-1}\right) \\
(3)\end{array}$ & $\begin{array}{c}c z_{\odot, \text { optical }} \\
\left(\mathrm{km} \mathrm{s}^{-1}\right) \\
(4)\end{array}$ & $\begin{array}{c}\text { NED Type } \\
(5)\end{array}$ & $\begin{array}{c}d_{C 1} \\
(\mathrm{kpc}) \\
(6)\end{array}$ & $\begin{array}{l}\text { HI ref. } \\
\text { (7) }\end{array}$ & $\begin{array}{c}\text { Optical ref. } \\
\text { (8) }\end{array}$ \\
\hline SDSS J120527.06+123243.2 & $120527.1,+123243$ & & 771 & $\operatorname{Im}$ & 297 & & AM06 \\
\hline SDSS J120640.69+120204.3 & $120640.7,+120204$ & & 1428 & & 152 & & AM06 \\
\hline AGC 226030 & $120820.7,+123004$ & 2320 & & & 171 & G07 & \\
\hline SDSS J120859.92+115631.2 & $120859.9,+115631$ & & & & 18 & & \\
\hline AGC 224602 & $121003.3,+114249$ & 2557 & 2594 & & 111 & K08 & AM06 \\
\hline VCC 20 & $121018.8,+121949$ & & & & 163 & & \\
\hline VCC 24 & $121035.7,+114538$ & 1296 & 1289 & $\mathrm{BCD}$ & 139 & K08 & F99 \\
\hline VCC 32 & $121102.7,+120615$ & & 1894 & $\mathrm{E}$ & 171 & & B85 \\
\hline VCC 35 & $121119.9,+115437$ & & & & 183 & & \\
\hline SDSS J121146.77+122938.3 & $121146.8,+122938$ & & 667 & & 272 & & AM06 \\
\hline VCC 41 & $121204.4,+124408$ & 2203 & & IB & 335 & B93 & \\
\hline VCC 46 & $121210.9,+125335$ & & 1437 & dE3 & 374 & & AM06 \\
\hline VCC 48 & $121215.0,+122913$ & 8 & -53 & & 298 & G07 & B85 \\
\hline VCC 58 & $121232.3,+120723$ & 2209 & 2213 & $\mathrm{SA}(\mathrm{rs}) \mathrm{bc}$ & 276 & G07 & B85;R99 \\
\hline IC 3041 & $121242.7,+124546$ & 1740 & 1738 & & 273 & G07 & AM06 \\
\hline VCC 65 & $121243.2,+120719$ & & & $\mathrm{dE}$ & 288 & & \\
\hline
\end{tabular}

Note.- Col. (1): Galaxy Name. Col. (2): RA and Decl. reported in NED. Col. (3): Heliocentric velocity based on HI measurements. Col. (4): Heliocentric velocity based on optical spectroscopy. Col. (5): NED morphological type. Col. (6): Projected linear displacement from Cloud 1 at the Virgo distance of $16.7 \mathrm{Mpc}$. Col. (7): HI measurement reference. Col. (8): Optical measurement reference. References are abbreviated as: G07: Giovanelli et al. 2007; K08: Kent et al. 2008; B85: Binggeli et al. 1985; B93: Binggeli et al. 1993; AM06: Adelman-McCarthy et al. 2006; F99: Falco et al. 1999 ; R99: Rubin et al. 1999 
TABLE 6

Galaxy Environment of Cloud $2\left(c z_{\odot}=2235 \mathrm{~km} \mathrm{~s}^{-1}\right)$

\begin{tabular}{|c|c|c|c|c|c|c|c|}
\hline $\begin{array}{c}\text { Galaxy Name } \\
\text { (1) }\end{array}$ & $\begin{array}{c}(\alpha, \delta) \\
(\mathrm{J} 2000) \\
(2)\end{array}$ & $\begin{array}{c}c z \odot, H I \\
\left(\mathrm{~km} \mathrm{~s}^{-1}\right) \\
(3)\end{array}$ & $\begin{array}{c}c z_{\odot, \text { optical }} \\
\left(\mathrm{km} \mathrm{s}^{-1}\right) \\
(4)\end{array}$ & $\begin{array}{c}\text { NED Type } \\
(5)\end{array}$ & $\begin{array}{c}d_{C 2} \\
(\mathrm{kpc}) \\
(6)\end{array}$ & $\begin{array}{l}\text { HI ref. } \\
\text { (7) }\end{array}$ & $\begin{array}{c}\text { Optical ref } \\
\text { (8) }\end{array}$ \\
\hline VCC 13 & $120946.3,+133301$ & & & & 337 & & \\
\hline VCC 15 & $120954.5,+130258$ & 2535 & 2505 & $\mathrm{Sm}$ & 273 & G07 & F99 \\
\hline VCC 20 & $121018.8,+121949$ & & & & 292 & & \\
\hline VCC 22 & $121024.2,+131014$ & 1699 & 1726 & $\mathrm{BCD}$ & 247 & G07 & AM06 \\
\hline VCC 23 & $121025.3,+132155$ & & & & 269 & & \\
\hline AGC 224696 & $121038.0,+130119$ & 2394 & 2418 & & 220 & G07 & AM06 \\
\hline VCC 32 & $121102.7,+120615$ & & 1894 & $\mathrm{E}$ & 298 & & B85 \\
\hline VCC 35 & $121119.9,+115437$ & & & & 333 & & \\
\hline VCC 36 & $121128.2,+133501$ & & & & 255 & & \\
\hline SDSS J121140.32+125824.6 & $121140.3,+125825$ & & 2221 & & 145 & & AM06 \\
\hline SDSS J121141.87+131146.7 & $121141.9,+131147$ & & 1496 & & 166 & & AM06 \\
\hline SDSS J121145.94+131707.9 & $121145.9,+131708$ & & 2468 & & 178 & & AM06 \\
\hline VCC 37 & $121146.2,+130124$ & & 2308 & dE5 & 142 & & AM06 \\
\hline SDSS J121153.85+134830.2 & $121153.9,+134830$ & & 2007 & & 294 & & AM06 \\
\hline VCC 41 & $121204.4,+124408$ & 2203 & & IB & 125 & B93 & \\
\hline VCC 46 & $121210.9,+125335$ & & 1437 & $\mathrm{dE} 3$ & 108 & & AM06 \\
\hline VCC 47 & $121211.7,+131446$ & 1875 & 1890 & $\mathrm{SAB}(\mathrm{r}) \mathrm{a}$ & 147 & G07 & AM06 \\
\hline VCC 49 & $121217.2,+131218$ & & 2295 & $\mathrm{E} 2$ & 134 & & AM06 \\
\hline VCC 55 & $121227.0,+131649$ & & & & 142 & & \\
\hline VCC 58 & $121232.3,+120723$ & 2209 & 2213 & $\mathrm{SA}(\mathrm{rs}) \mathrm{bc}$ & 240 & G07 & B85 \\
\hline IC 3041 & $121242.7,+124546$ & 1740 & 1738 & & 80 & G07 & AM06 \\
\hline VCC 65 & $121243.2,+120719$ & & & & 236 & & \\
\hline VCC 68 & $121249.0,+132050$ & & 2425 & & 145 & & AM06 \\
\hline VCC 70 & $121256.4,+130407$ & & & & 73 & & \\
\hline AGC 224807 & $121309.4,+133504$ & 2100 & 2108 & & 204 & G07 & AM06 \\
\hline SDSS J121313.69+133122.0 & $121313.7,+133122$ & & 2158 & & 185 & & AM06 \\
\hline SDSS J121317.79+130935.6 & $121317.8,+130936$ & & 1915 & & 82 & & AM06 \\
\hline VCC 84 & $121335.3,+132413$ & & & & 148 & & \\
\hline VCC 85 & $121336.4,+130201$ & 1932 & & & 40 & $\mathrm{Ga06}$ & \\
\hline VCC 89 & $121347.3,+132528$ & 2114 & 2115 & $\mathrm{SAB}(\mathrm{rs}) \mathrm{cd}$ & 154 & G07 & AM06 \\
\hline VCC 97 & $121353.6,+131021$ & 2470 & 2480 & $\mathrm{SAB}(\mathrm{s}) \mathrm{c}$ & 81 & G07 & F95 \\
\hline VCC 98 & $121353.8,+135213$ & & & & 284 & & \\
\hline VCC 100 & $121404.0,+133908$ & & & & 222 & & \\
\hline VCC 106 & $121409.0,+115619$ & & & & 281 & & \\
\hline VCC 107 & $121410.7,+131407$ & & & & 104 & & \\
\hline SDSS J121419.86+132706.4 & $121419.9,+132706$ & & 2467 & & 168 & & AM06 \\
\hline VCC 113 & $121432.8,+120611$ & 2115 & 2139 & & 239 & G07 & AM06 \\
\hline VCC 122 & $121444.2,+121048$ & & 2348 & So & 222 & & AM06 \\
\hline AGC 224705 & $121444.6,+124723$ & 2279 & 2298 & & 81 & G07 & AM06 \\
\hline VCC 132 & $121503.8,+130155$ & 2085 & & SB & 105 & G07 & \\
\hline VCC 133 & $121505.2,+130644$ & & & & 117 & & \\
\hline VCC 135 & $121506.8,+120058$ & 2402 & 2412 & $\mathrm{Sa}$ & 276 & S05 & AM06 \\
\hline VCC 146 & $121520.8,+123656$ & & & & 143 & & \\
\hline VCC 150 & $121528.6,+123856$ & & & & 146 & & \\
\hline VCC 155 & $121535.7,+133711$ & & & & 250 & & \\
\hline VCC 163 & $121546.0,+123344$ & & & & 177 & & \\
\hline VCC 164 & $121552.6,+120150$ & & & & 296 & & \\
\hline VCC 175 & $121602.8,+123544$ & & & & 189 & & \\
\hline VCC 185 & $121620.1,+130814$ & & & & 200 & & \\
\hline VCC 197 & $121632.7,+130944$ & & & & 216 & & \\
\hline VCC 204 & $121639.2,+125220$ & & & & 210 & & \\
\hline VCC 215 & $121658.3,+121549$ & & 2074 & $\mathrm{dE} 4$ & 297 & & AM06 \\
\hline VCC 224 & $121709.2,+122712$ & 2131 & 2109 & Sbc & 278 & G07 & AM06 \\
\hline VCC 230 & $121719.5,+115632$ & & 1429 & $\mathrm{dE} 4$ & 380 & & AM06 \\
\hline VCC 232 & $121723.6,+133020$ & & & & 316 & & \\
\hline AGC 224489 & $121728.1,+125556$ & 2056 & 2080 & & 268 & G07 & AM06 \\
\hline SDSS J121731.31+115715.9 & $121731.3,+115716$ & & & & 387 & & \\
\hline VCC 241 & $121733.9,+122320$ & & & & 312 & & \\
\hline
\end{tabular}

Note.- Col. (1): Galaxy Name. Col. (2): RA and Decl. reported in NED. Col. (3): Heliocentric velocity based on HI measurements. Col. (4): Heliocentric velocity based on optical spectroscopy. Col. (5): NED morphological type. Col. (6): Projected linear displacement from Cloud 2 at the Virgo distance of $16.7 \mathrm{Mpc}$. Col. (7): HI measurement reference. Col. (8): Optical measurement reference. References are abbreviated as: G07: Giovanelli et al. 2007; K08: Kent et al. 2008; Ga06: Gavazzi et al. 2006; B85: Binggeli et al. 1985; B93: Binggeli et al. 1993; AM06: Adelman-McCarthy et al. 2006; F99: Falco et al. 1999; R99: Rubin et al. 1999; T08: Tully et al. 2008; S05: Springob et al. 2005b; F95: Fisher et al. 1995 

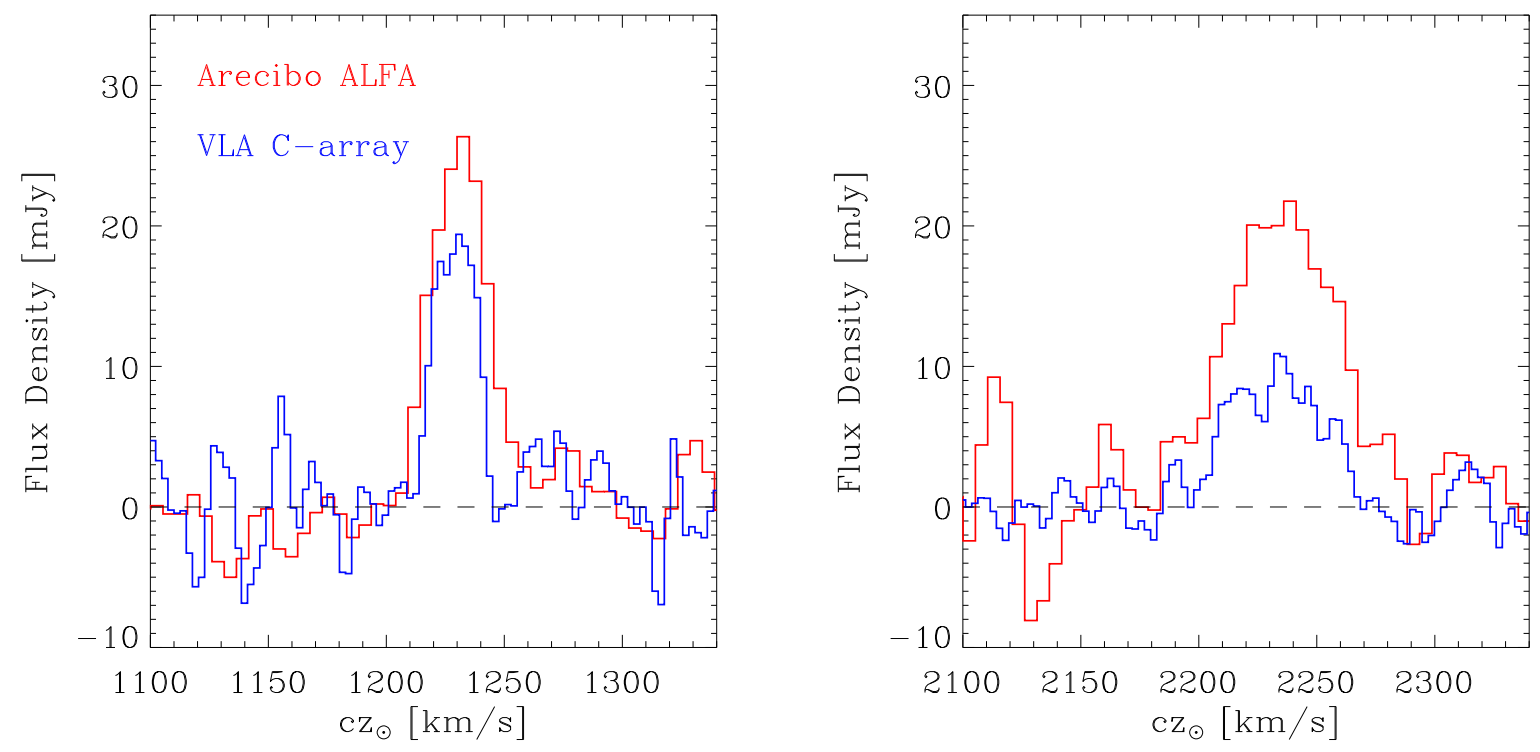

Fig. 1.- Integrated spectral profiles of the Cloud 1 (left) and Cloud 2 (right) detections made with Arecibo and the ALFALFA survey (red), and total integrated profiles from the multiple cloud detections in the same fields made with the VLA in C configuration (blue). The channel resolution of the VLA spectrum is $12.2 \mathrm{kHz}\left(2.6 \mathrm{~km} \mathrm{~s}^{-1}\right)$. The global profiles for the VLA were obtained by summing all emission for $N_{H I}^{\prime} \geq 1.5 \times 10^{20} \mathrm{~cm}^{-2}$ in Figure 4 and for $N_{H I}^{\prime} \geq 0.7 \times 10^{20} \mathrm{~cm}^{-2}$ in Figure 5 , 


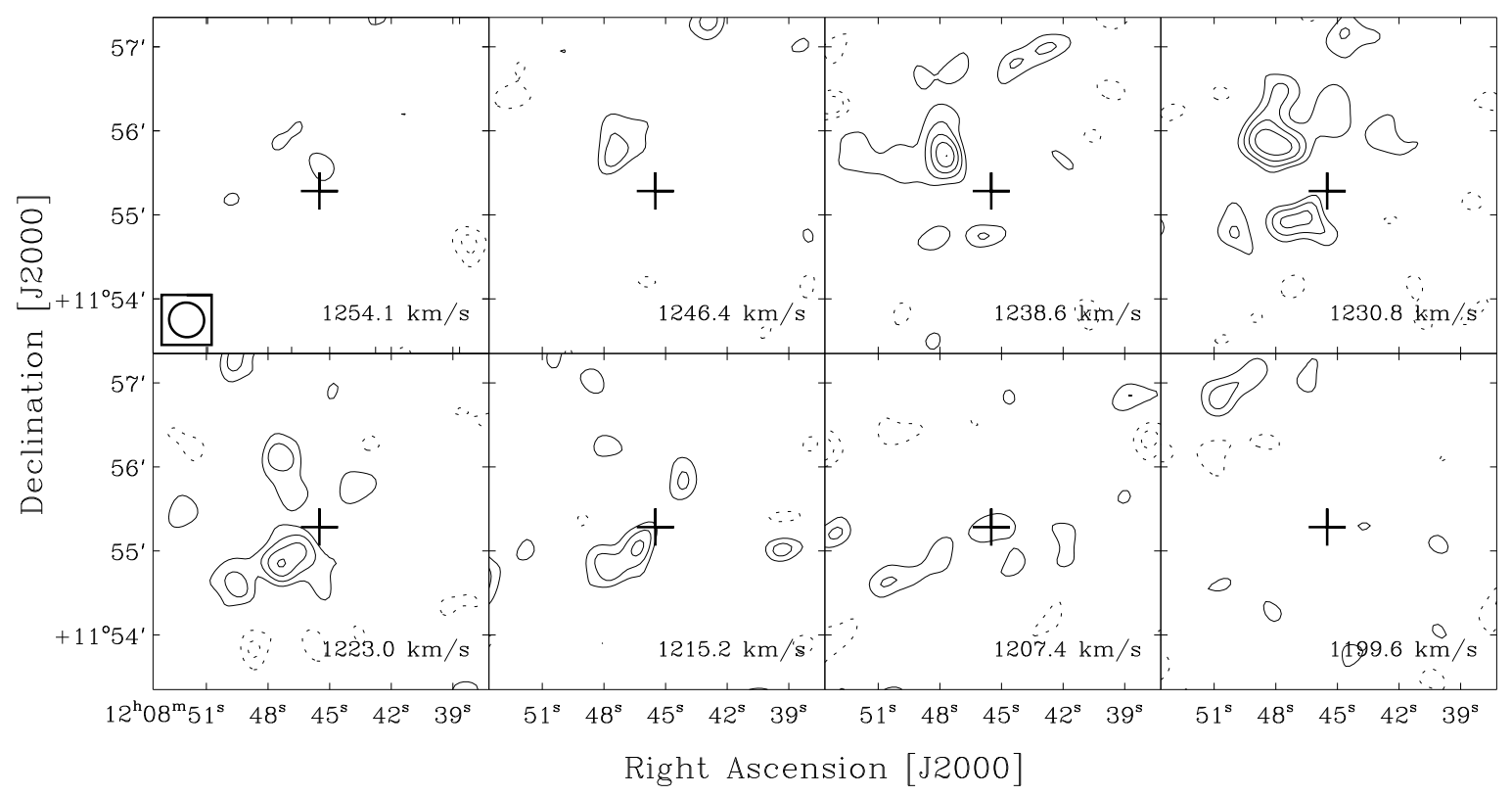

Fig. 2.- Naturally-weighted channel maps for the Cloud 1 field from the VLA-C observations. The plotted channels are independent $\left(\delta V^{\prime}=7.8 \mathrm{~km} \mathrm{~s}^{-1}\right)$. Contours are at $\left(-3,-2,2\left(2 \sigma_{m}^{\prime}\right), 3,4,5,6\right) \mathrm{mJy} /$ beam; negative contours are represented with dashed lines. The cross indicates the centroid position of the original Cloud 1 Arecibo detection (Table 4). The heliocentric radial velocity is in the lower right corner of each panel, and the synthesized beam is in the lower left corner of the first panel. 


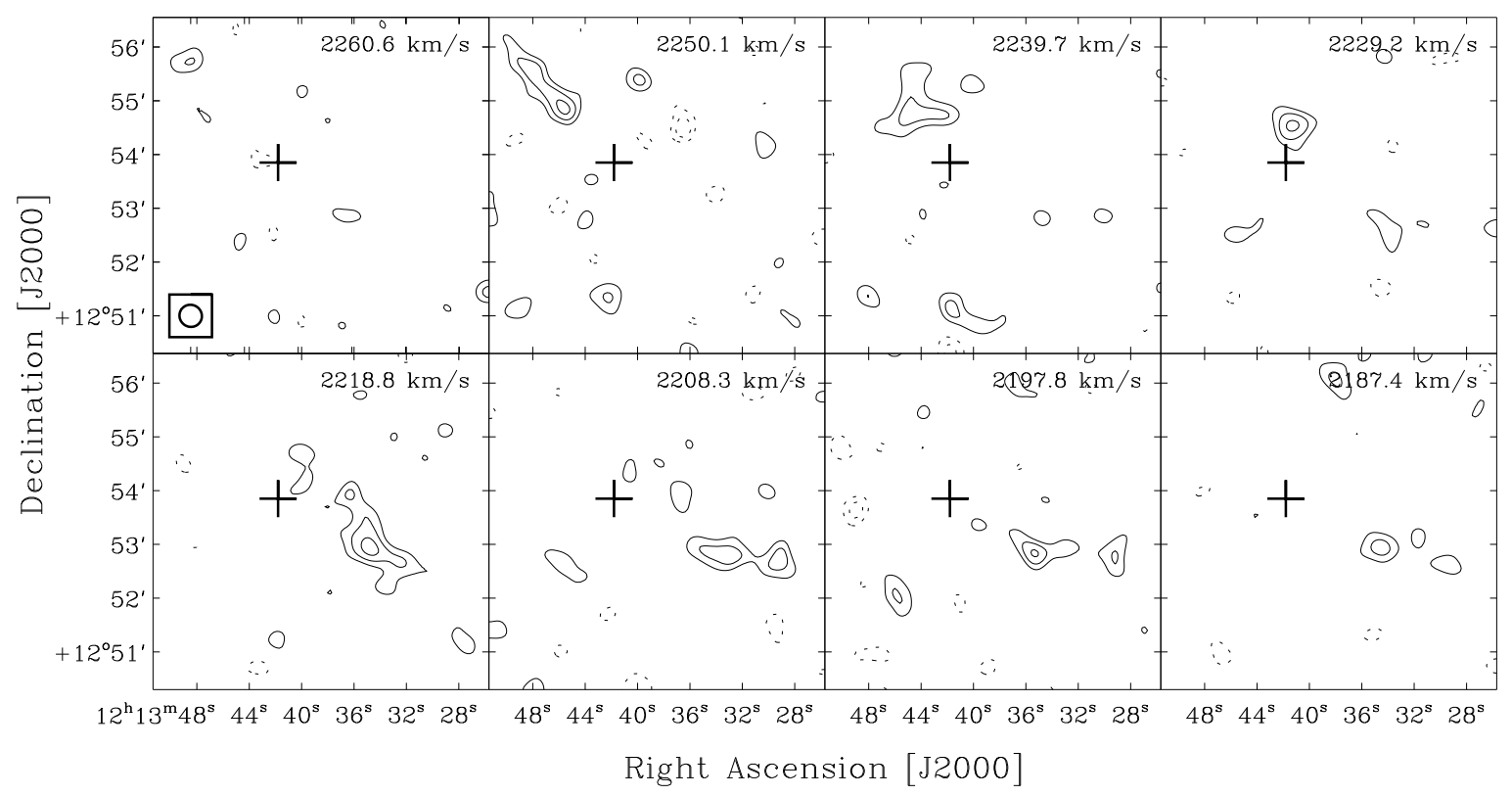

Fig. 3.- Naturally-weighted channel maps for the Cloud 2 field from the VLA-C observations. The plotted channels are independent $\left(\delta V^{\prime}=10.4 \mathrm{~km} \mathrm{~s}^{-1}\right)$. Contours are at $\left(-3,-2,2\left(2 \sigma_{m}^{\prime}\right), 3,4,5,6\right) \mathrm{mJy} /$ beam; negative contours are represented with dashed lines. The cross indicates the centroid position of the original Cloud 2 Arecibo detection (Table 4). The heliocentric radial velocity is in the upper right corner of each panel, and the synthesized beam is in the lower left corner of the first panel. 


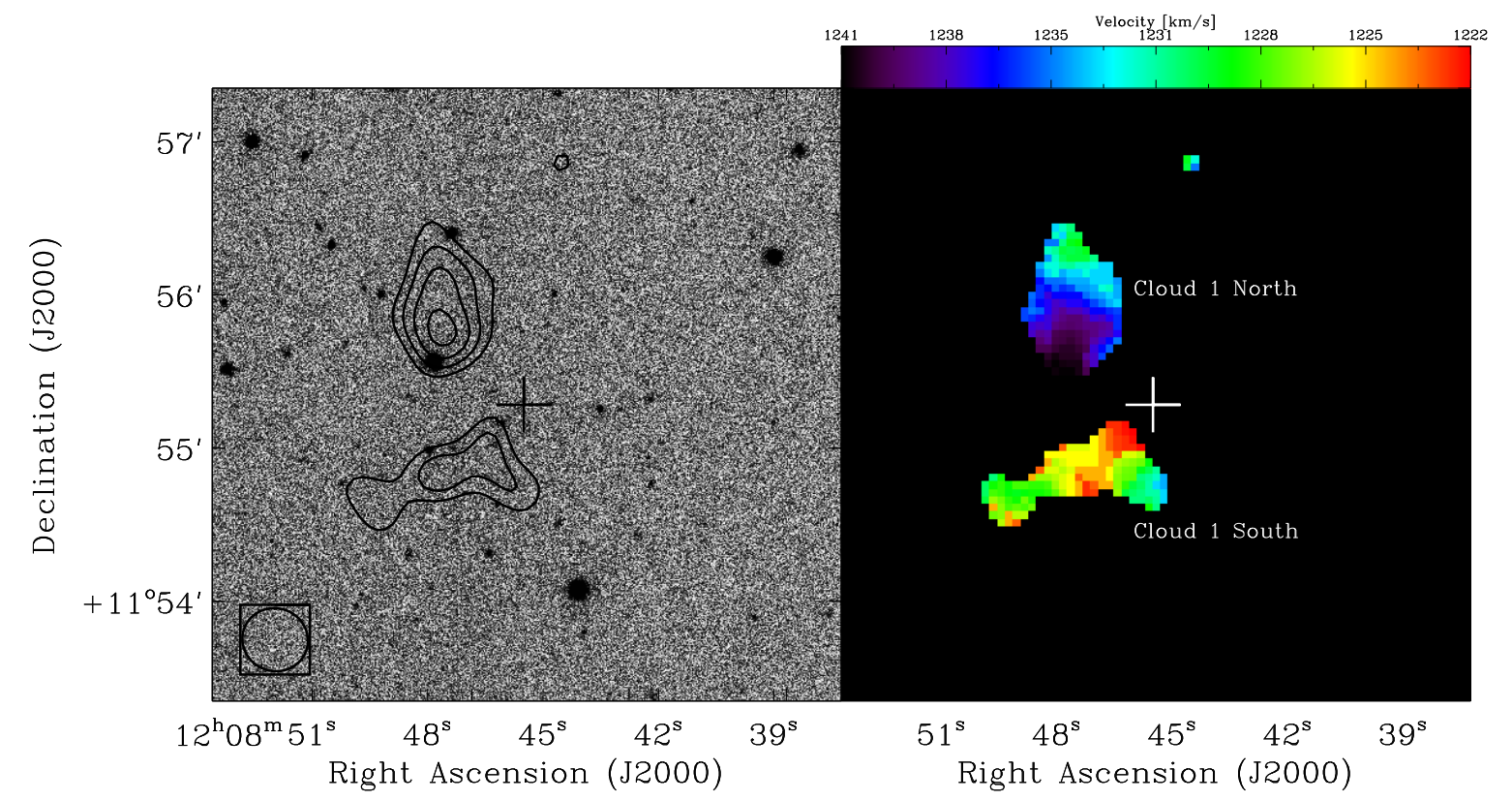

Fig. 4.- H I distribution and kinematics of the Cloud 1 Field, showing Cloud 1 North and 1 South as detected in the VLA-C data cube. The left panel shows total intensity map of the clouds (contours) superimposed on an SDSS $g$-band image (grayscale). Contours are at $N_{H I}^{\prime}=10^{20} \times(1.5,2,2.5,3) \mathrm{cm}^{-2}$, and the grayscale is plotted logarithmically. The cross indicates the centroid position of the original Cloud 1 Arecibo detection (Table 4). The synthesized beam is in the lower left corner of the panel. The right panel shows intensity-weighted velocity map of the clouds in regions where $N_{H I}^{\prime} \geq 1.5 \times 10^{20} \mathrm{~cm}^{-2}$. The velocity spans $1222-1241 \mathrm{~km} \mathrm{~s}^{-1}$ on a linear scale, as indicated by the colorbar at the top of the plot. 


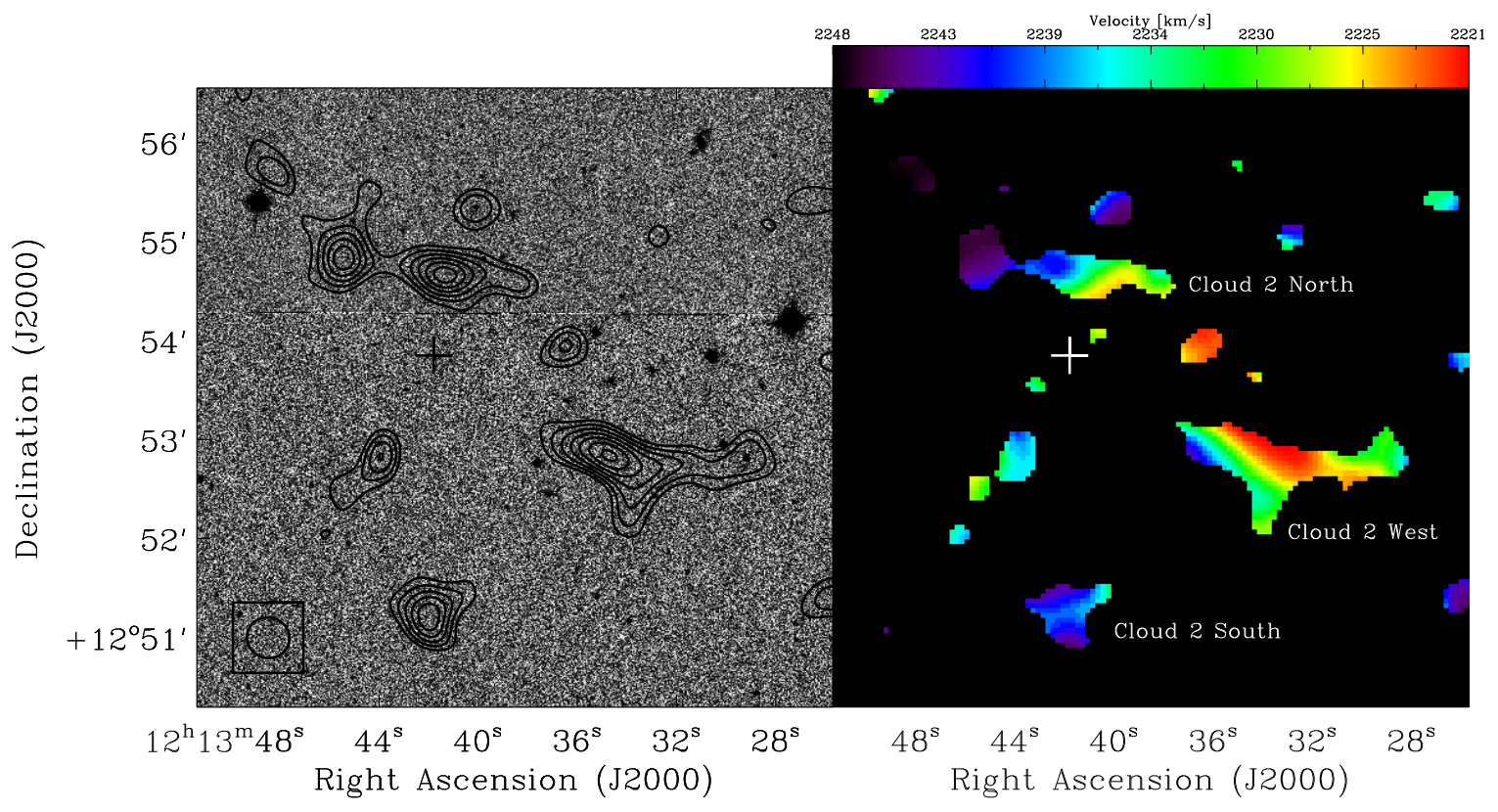

Fig. 5.- H I distribution and kinematics of the Cloud 2 Field. The left panel shows total intensity map of the clouds (contours) superimposed on an SDSS $g$-band image (grayscale). Contours are at $N_{H I}^{\prime}=10^{20} \times(0.7,0.8,0.9,1.0,1.1,1.2,1.3,1.4,1.5) \mathrm{cm}^{-2}$, and the grayscale is plotted logarithmically. The cross indicates the centroid position of the original Cloud 2 Arecibo detection (Table 4). The synthesized beam is in the lower left corner of the panel. The right panel shows intensity-weighted velocity map of the clouds in regions where $N_{H I}^{\prime} \geq 1.5 \times 10^{20} \mathrm{~cm}^{-2}$. The velocity spans $2222-2248 \mathrm{~km} \mathrm{~s}^{-1}$ on a linear scale, as indicated by the colorbar at the top of the plot. 

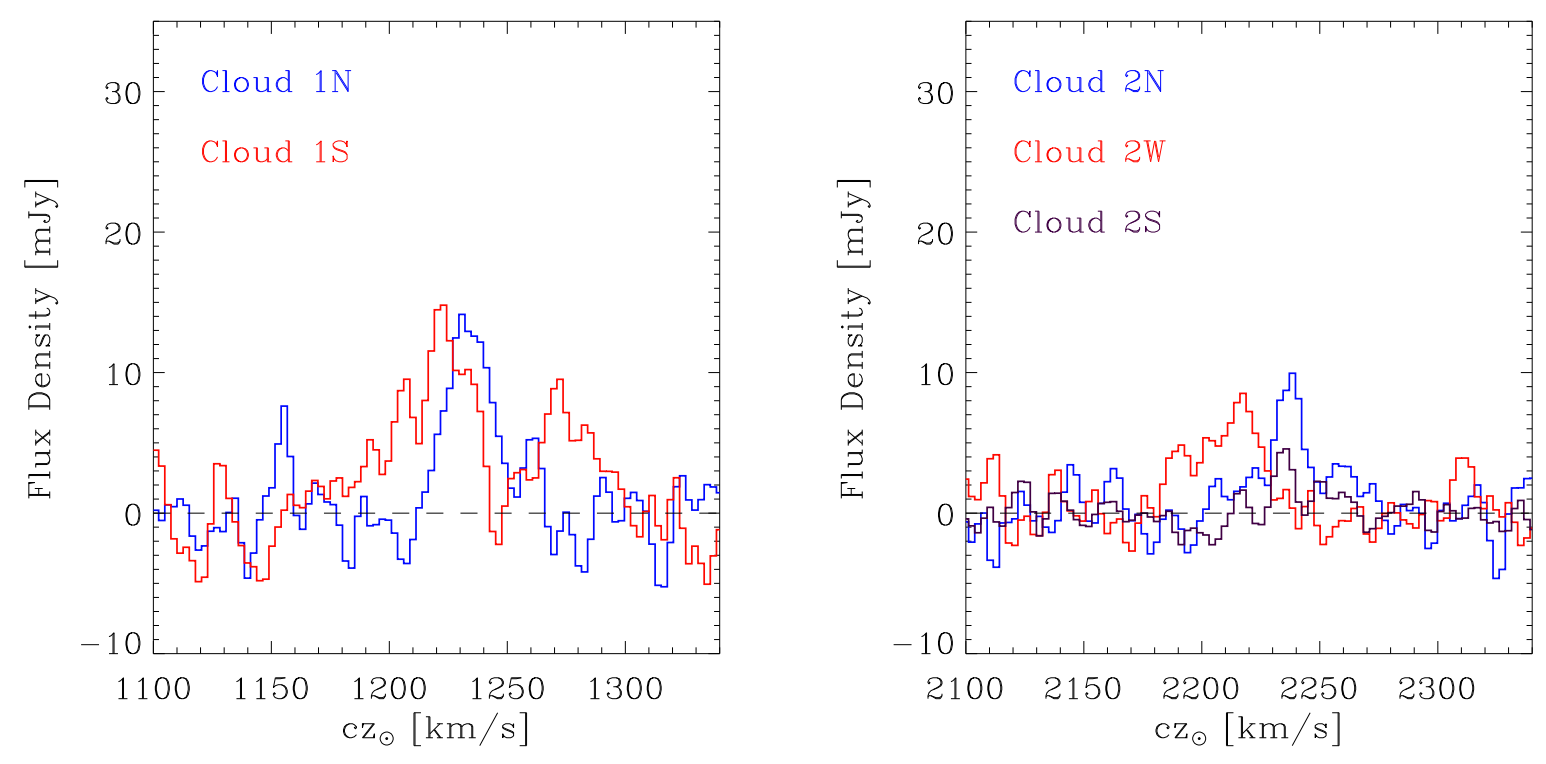

Fig. 6.- Integrated spectral profiles of VLA-C detections in the Cloud 1 field (left) and Cloud 2 field (right). 


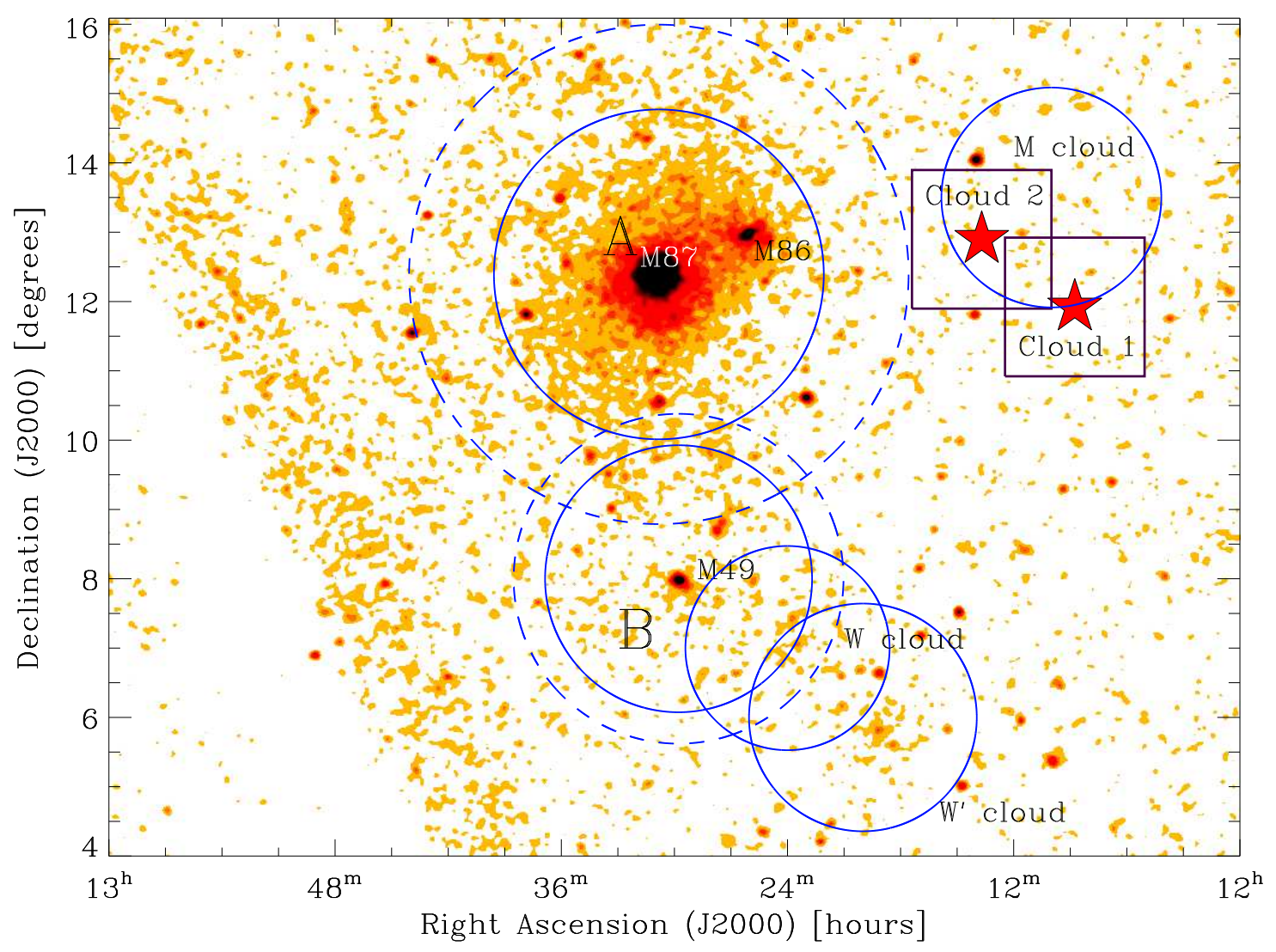

Fig. 7.- The location of the two Arecibo HI cloud detections in the context of the greater cluster environment. The centroid positions of Cloud 1 and Cloud 2 are indicated by star symbols. The peaks from the hard X-ray background image provided by ROSAT are labeled indicating Virgo cluster galaxies M49 and M87 (Snowden et al. 1995). The symbols are not indicative of source sizes and are shown only for positional indication. The dashed lines indicate the projected virial radius of the dark matter halo $r_{200} / 2$ determined by McLaughlin (1999), for the A and B areas centered around cluster members M87 and M49 respectively. The two $2^{\circ}$ boxes surrounding the detections show the areas of sky examined in Figure 8 . 

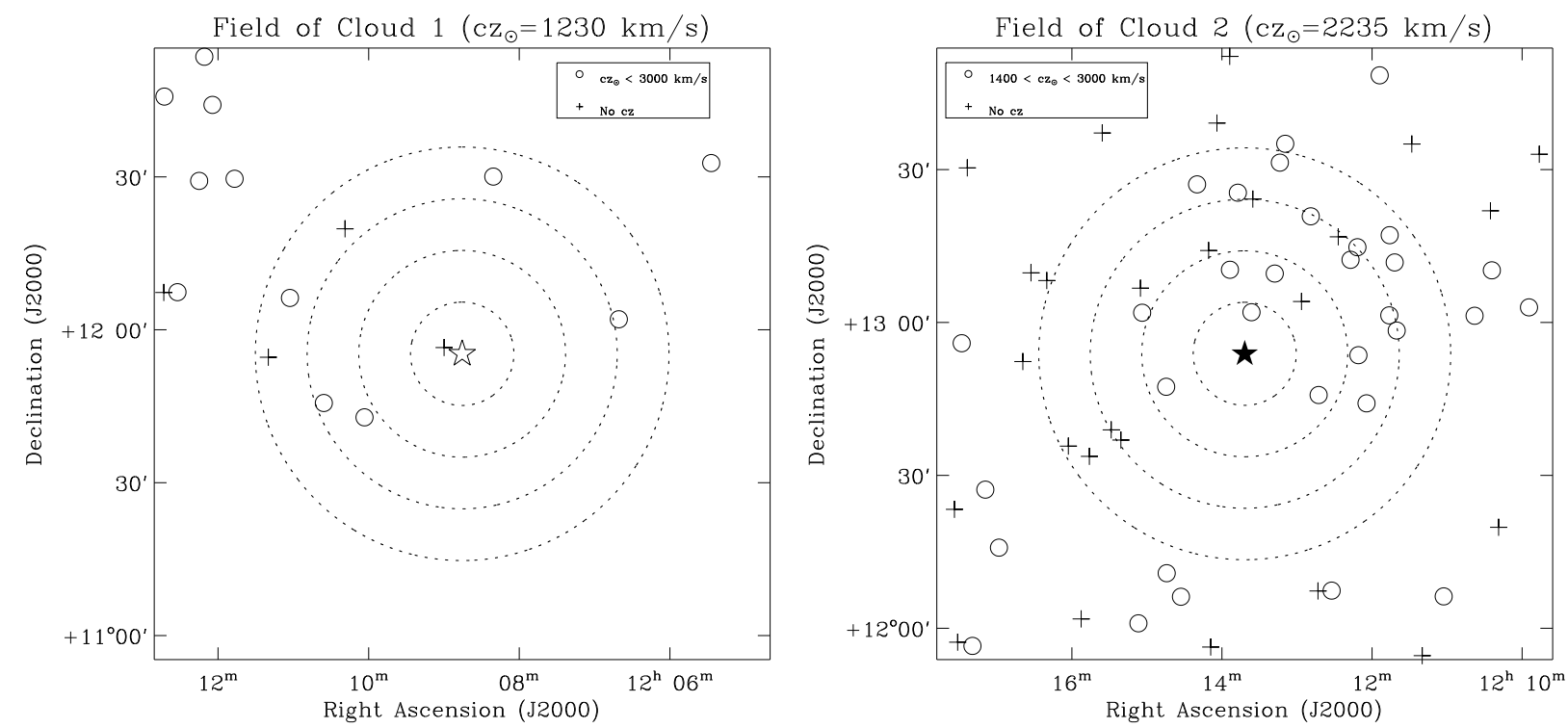

Fig. 8.- Galaxies in the vicinity of the two cloud complexes. The plots show $2^{\circ} \times 2^{\circ}$ areas of sky surrounding the Arecibo detection Cloud 1 (left; white star), and Cloud 2 (right; dark star). The plot shows all objects with published redshifts of $c z_{\odot}<3000 \mathrm{~km} \mathrm{~s}^{-1}$ for the Cloud 1 area and $1400<c z_{\odot}<3000 \mathrm{~km} \mathrm{~s}^{-1}$ for the Cloud 2 area. The crosses indicate galaxies without published redshifts. The dashed circles are of radii 50, 100, 150 and $200 \mathrm{kpc}$ projected on the sky at the Virgo Cluster distance of $16.7 \mathrm{Mpc}$. 